\title{
Design of metamaterial mechanisms using robust topology optimization and variable linking scheme
}

\author{
Wu, Kai; Sigmund, Ole; Du, Jianbin
}

Published in:

Structural and Multidisciplinary Optimization

Link to article, DOI:

10.1007/s00158-020-02791-1

Publication date:

2021

Document Version

Peer reviewed version

Link back to DTU Orbit

Citation (APA):

Wu, K., Sigmund, O., \& Du, J. (2021). Design of metamaterial mechanisms using robust topology optimization and variable linking scheme. Structural and Multidisciplinary Optimization, 63, 1975-1988.

https://doi.org/10.1007/s00158-020-02791-1

\section{General rights}

Copyright and moral rights for the publications made accessible in the public portal are retained by the authors and/or other copyright owners and it is a condition of accessing publications that users recognise and abide by the legal requirements associated with these rights.

- Users may download and print one copy of any publication from the public portal for the purpose of private study or research.

- You may not further distribute the material or use it for any profit-making activity or commercial gain

- You may freely distribute the URL identifying the publication in the public portal

If you believe that this document breaches copyright please contact us providing details, and we will remove access to the work immediately and investigate your claim 


\title{
Design of Metamaterial Mechanisms using Robust Topology Optimization and Variable Linking Scheme
}

\author{
Kai Wu${ }^{1}$, Ole Sigmund ${ }^{2}$, Jianbin $\mathrm{Du}^{3^{*}}$ \\ ${ }^{1}$ School of Aerospace Engineering, Tsinghua University, \\ Beijing, 100084, China \\ e-mail: k.wu-4@tudelft.nl \\ ${ }^{2}$ Department of Mechanical Engineering, Technical University of Denmark, \\ Building 404, DK-2800 Lyngby, Denmark \\ e-mail: sigmund@mek.dtu.dk \\ ${ }^{3}$ School of Aerospace Engineering, Tsinghua University, \\ Beijing, 100084, China \\ e-mail: dujb@tsinghua.edu.cn \\ *Corresponding author
}

\begin{abstract}
Metamaterial mechanisms are structures composed of periodic cells that possess special mechanism responses. This paper proposes a topology optimization method based on a variable linking scheme for the design of metamaterial mechanisms. A robust formulation is included to improve the manufacturing reliability of the designs and prevent de-facto hinges. The proposed method is applied to the designs of a force inverter and some other interesting mechanisms. Numerical examples illustrate that metamaterial structures with desired mechanism performances can be obtained by the presented method. Studies are performed to compare the responses and robustness between conventional compliant mechanisms and metamaterial mechanisms. Furthermore, multiple subdomains are adopted to introduce more flexibility in the design. The work highlights the pros and cons of metamaterial mechanisms compared to conventional compliant mechanisms.
\end{abstract}

Keywords Metamaterial mechanisms; Variable linking scheme; Topology optimization; Robust design.

\section{Introduction}

Topology optimization is now one of the most important methods for structural optimization and has a wide range of applications in different areas (Eschenauer and Olhoff 2001; Xu and Cheng 2010).

Mechanical metamaterials are artificial structures with mechanical properties that are defined by their repetitive cell patterns rather than the base material they are made of. There have been many studies on metamaterials, such as negative Poisson's ratio structures (Lakes 1987; Milton 1992), metamaterials with negative stiffness (Lakes et al. 2001), metamaterials with negative compressibility (Nicolaou and Motter 2012), metamaterials with programmable mechanical properties (Florijn et al. 2014), high strength metamaterials (Bauer et al. 2016) and metamaterials with coupling effects between tension/compression (Frenzel et al. 2017).

Topology optimization can be an efficient tool for the design of mechanical metamaterials. Sigmund (1994) obtained negative Poisson's ratio metamaterials by inverse homogenization method. Clausen et al. (2015) fabricated a new class of 
architected materials with programmable Poisson's ratio over large deformations. Wang (2018) designed 3D auxetic lattice materials which exhibit constant negative Poisson's ratios over large strain intervals. Chen and Huang (2019) designed chiral metamaterial with coupling effects between torsion and compression.

Aside from mechanical metamaterials, topology optimization can also be used to design acoustic, thermal, optical or multiphysics metamaterials, e.g. metamaterials with negative thermal expansion coefficient (Sigmund and Torquato 1996), metamaterials with negative permeability (Diaz and Sigmund 2009), acoustic metamaterials with negative bulk modulus (Lu et al. 2013), acoustic metamaterials exhibiting negative refraction with tunable high transmission (Christiansen and Sigmund 2016a; Christiansen and Sigmund 2016b) and acoustic topological insulators (Christiansen et al. 2019).

Another important field for the application of structural topology optimization is the synthesis of compliant mechanisms, which are devices that can transform input forces and motion into a desired set of output forces and motion, e.g. force inverters or gripping structures (Sigmund 1997).

Since the first paper by Ananthasuresh et al. (1994), topology optimization has become an important design tool for compliant mechanisms (Zhu et al. 2020). A common issue in the design of compliant mechanisms is to eliminate one-node connected hinges, which takes advantage of flaws in finite element modeling to create de-facto hinges. There are several ways to resolve this, for example, introducing stress constraints (da Silva et al. 2019; De Leon et al. 2015), multiscale wavelet based topology optimization (Yoon et al. 2004), strain-based multi-objective formulation (Lee and Gea 2014) or robust design formulation (Sigmund 2009).

Metamaterial mechanisms are the combination of the above two concepts, referring to structures with special mechanism responses, which are partly or wholly composed of rationally designed periodic cells. The concept has been proposed by Ion et al. (2016), who created some newfangled macroscopic mechanisms from periodic unit cells. In their paper, metamaterial mechanisms are understood as 'machines' combined of finite macroscopic periodic cells to achieve desired mechanical performances, which is quite different from metamaterial design using inverse homogenization. For example, Fig. 1 shows a plier and a door latch implemented as metamaterial mechanisms from the paper by Ion et al. (2016).

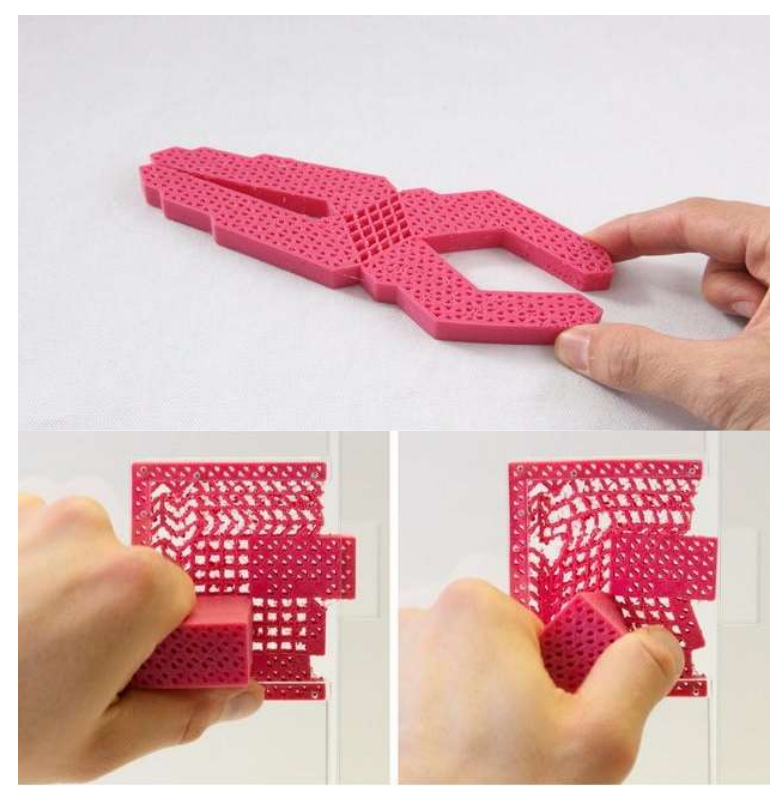

Fig. 1 Typical metamaterial mechanisms designed by Ion et al. (2016).

However, in Ion's paper, they did not propose any universal systematic method for designing metamaterial mechanisms. On this basis, the present paper aims at proposing a robust topology optimization approach based on a variable linking scheme for the design of metamaterial mechanisms, and further analyze their pros and cons compared to conventional compliant mechanisms.

This paper is organized as follows: Section 2 defines the basic problem of metamaterial mechanism design based on topology optimization. In Section 3, the mathematical model of topology optimization based on a variable linking scheme, including the 
sensitivity analysis and the three-field robust formulation, is presented and discussed in detail. Numerical examples are given in Section 4 to validate the proposed method. Some interesting features are revealed and discussed as well. Structural robustness and stress analyses are performed in Section 5 to make further comparisons between metamaterial mechanisms and conventional compliant mechanisms. Finally, some conclusions are given in Section 6.

\section{Problem Definition}

The design domain for metamaterial mechanisms is similar to that of conventional compliant mechanisms. As can be seen in Fig. 2 , the domain is subjected to an input force $f_{\text {in }}$ and an input spring $k_{\text {in }}$ at the input point, modelling a strain-based actuator with blocking force $F_{\text {in }}$. The actuator causes an output displacement $u_{\text {out }}$ or force $f_{\text {out }}$ on the workpiece at the output, which is simplified as an output spring $k_{\text {out }}$. The whole design domain is discretized by finite elements.

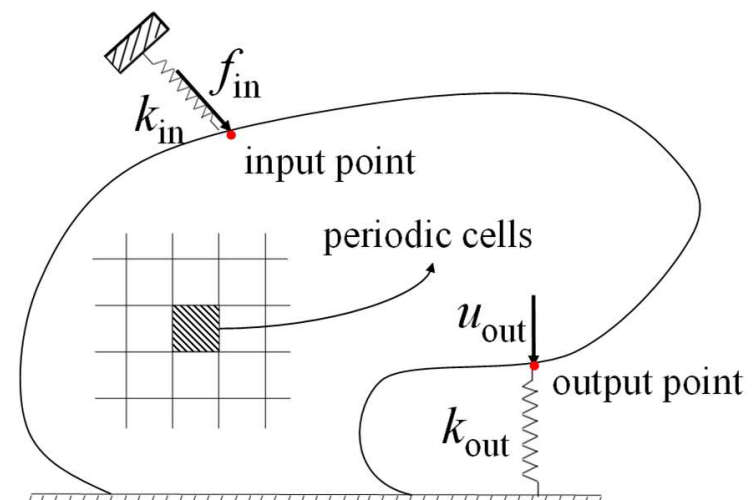

Fig. 2 Design domain and boundary conditions for metamaterial mechanism design using a variable linking scheme.

In the design of conventional compliant mechanisms, a relative density $\rho$ will be assigned to each element as design variable (see e.g. Bendsoe and Sigmund 2004). In the design of metamaterial mechanisms using a variable linking scheme, the domain is further divided into periodic cells, and the design variables are defined only on one of these cells (e.g. the shaded one) and then copied to the others.
It should be noted that the scale of those periodic cells is comparable to that of the macro domain. The proposed variable linking topology optimization is hence in practice a single scale method executed on the structural level.

\section{Topology Optimization Based on Variable linking scheme}

\subsection{Optimization model}

A simple way to do variable linking on structured grids is by defining a sparse mapping matrix $\boldsymbol{G}$. The design variable $\kappa_{i}$ is defined on each finite element in the design cell, and the linked variables $\rho$ of elements in other cells can be obtained by $\boldsymbol{\rho}=\boldsymbol{G} \boldsymbol{\kappa}$,

$$
\boldsymbol{G}=\left[\begin{array}{cccccc}
1 & 0 & 0 & \cdots & 0 & \cdots \\
0 & 1 & 0 & \cdots & 0 & \cdots \\
\vdots & \vdots & \vdots & \vdots & \vdots & \vdots \\
0 & 0 & \cdots & 1 & 0 & \cdots \\
1 & 0 & 0 & \cdots & 0 & \cdots \\
0 & 1 & 0 & \cdots & 0 & \cdots \\
\vdots & \vdots & \vdots & \vdots & \vdots & \vdots \\
\vdots & \vdots & \vdots & \vdots & \vdots & \vdots \\
0 & 0 & \cdots & 0 & 1 & 0 \\
0 & 0 & \cdots & 0 & 0 & 1
\end{array}\right]_{N_{\mathrm{e}} \times n_{\mathrm{e}}}
$$

$N_{\mathrm{e}}$ is the total number of finite elements in the whole design domain and $n_{\mathrm{e}}$ is the number of finite elements in the single design cell, that is the number of design variables. If the density of element $j$, i.e. $\rho_{j}$ is linked to the $i$-th design variable $\kappa_{i}$, then $\boldsymbol{G}(j, i)=1$.

The typical objective of the mechanism design is to maximize the displacements at some specified output points in specific directions. The general formulation can be written as: 


$$
\begin{aligned}
& \min _{\boldsymbol{\kappa}}\left\{f_{0}=\boldsymbol{L}^{\mathrm{T}} \boldsymbol{U}\right\} \\
& \text { s.t. } \boldsymbol{K} \boldsymbol{U}=\boldsymbol{P}, \\
& f_{1}=\frac{\boldsymbol{\rho}^{\mathrm{T}} \boldsymbol{V}}{V_{0}} \leq \frac{V^{*}}{V_{0}}, \\
& 0 \leq \boldsymbol{\kappa} \leq 1 .
\end{aligned}
$$

where $\boldsymbol{U}$ and $\boldsymbol{P}$ represent the vectors of the nodal displacements and loads, respectively. $\boldsymbol{L}$ is a selection vector selecting the specified output degrees of freedom. $\boldsymbol{V}$ denotes the element volume vector and the upper limit of the material usage is given as $V^{*}=\alpha V_{0}$, where $\alpha$ is the material volume fraction and $V_{0}$ is the total volume of the domain. $\boldsymbol{K}$ is the global stiffness matrix, which is assembled from individual element stiffness matrices as $\boldsymbol{K}_{j}=E_{j} \boldsymbol{K}_{0}$, where $\boldsymbol{K}_{0}$ is the element stiffness matrix for unit stiffness and $E_{j}$ is the material stiffness obtained by the Solid Isotropic Material Interpolation with Penalization (SIMP) (Bendsøe and Sigmund 1999) given as:

$$
E_{j}=E_{\min }+\rho_{j}{ }^{p}\left(E_{0}-E_{\min }\right)
$$

where $E_{0}$ is the stiffness of the solid phase, $p=3$ is the penalization power.

Using the variable linking scheme, the sensitivity of the objective and constraint functions with respect to the design variables should be calculated as:

$$
\begin{aligned}
& \frac{\partial f_{0}}{\partial \boldsymbol{\kappa}}=\boldsymbol{G}^{\mathrm{T}} \frac{\partial f_{0}}{\partial \boldsymbol{\rho}} \\
& \frac{\partial f_{1}}{\partial \boldsymbol{\kappa}}=\boldsymbol{G}^{\mathrm{T}} \frac{\partial f_{1}}{\partial \boldsymbol{\rho}}
\end{aligned}
$$

The sensitivity analysis is then conducted using the adjoint method as discussed later. The optimization algorithm used in this article is the Method of Moving Asymptotes (MMA) (Svanberg 1987). Analysis here is restricted to linear elasticity.

\subsection{Projection and filtering}

In order to solve discretization issues such as mesh dependence and checkerboards, the topology optimization model should be regularized.

In this paper, projection techniques are used to project the design variables to near 01 solution. In particular, smoothed threshold projection scheme based on the tanh function (Wang et al. 2011) is adopted:

$$
\overline{\tilde{\rho}}_{k}=\frac{\tanh (\beta \eta)+\tanh \left(\beta\left(\tilde{\rho}_{k}-\eta\right)\right)}{\tanh (\beta \eta)+\tanh (\beta(1-\eta))}
$$

where $\overline{\tilde{\rho}}_{k}$ is the physical density and $\tilde{\rho}_{k}$ is the filtered density as defined below. $\beta$ is a projection parameter which controls the sharpness of the smoothed projection function, $\eta$ refers to the threshold value. The derivative of the projected physical density $\overline{\tilde{\rho}}_{k}$ with respect to the filtered density $\tilde{\rho}_{k}$ is calculated as follows:

$\frac{\partial \overline{\tilde{\rho}}_{k}}{\partial \tilde{\rho}_{k}}=\frac{1-\tanh ^{2}\left(\beta\left(\tilde{\rho}_{k}-\eta\right)\right)}{\tanh (\beta \eta)+\tanh (\beta(1-\eta))} \beta$

The filtered density $\tilde{\rho}_{k}$ is calculated as:

$\tilde{\rho}_{k}=\frac{\sum_{j \in \mathbb{N}_{e, k}} w\left(\boldsymbol{x}_{j}\right) V_{j} \rho_{j}}{\sum_{j \in \mathbb{N}_{e, k}} w\left(\boldsymbol{x}_{j}\right) V_{j}}$

where $\mathbb{N}_{\mathrm{e}, k}$ is the neighbourhood set of elements within the filter radius $R$ of element $k, w(\boldsymbol{x})$ is the weight function defined as:

$w\left(\boldsymbol{x}_{j}\right)=R-\left|\boldsymbol{x}_{j}-\boldsymbol{x}_{k}\right|$

where $\boldsymbol{x}_{j}, \boldsymbol{x}_{k}$ refer to the central coordinates of elements $j, k$.

It should be noted that in the variable linking scheme, the filtering is still applied on the whole domain, instead of on single cell. The derivative of the filtered density $\tilde{\rho}_{k}$ with respect to $\rho_{j}$ is calculated as:

$$
\frac{\partial \tilde{\rho}_{k}}{\partial \rho_{j}}=\frac{w\left(\boldsymbol{x}_{j}\right) V_{j}}{\sum_{j \in \mathbb{N}_{\mathrm{e}, k}} w\left(\boldsymbol{x}_{j}\right) V_{j}}=\boldsymbol{H}(k, j)
$$

where $\boldsymbol{H}$ is a $N_{\mathrm{e}} \times N_{\mathrm{e}}$ sparse matrix, if 
element $j$ is not in the neighbourhood set of element $k, \boldsymbol{H}(k, j)=0$.

Considering filtering and projection, as well as standard adjoint method, the sensitivity of objective and constraint functions can be calculated as:

$$
\begin{aligned}
\frac{\partial f_{0}}{\partial \boldsymbol{\kappa}} & =\boldsymbol{G}^{\mathrm{T}} \boldsymbol{H}^{\mathrm{T}}\left[-\frac{\partial \overline{\tilde{\rho}}_{k}}{\partial \tilde{\rho}_{k}} p \overline{\tilde{\rho}}_{k}^{p-1} \lambda_{k}^{\mathrm{T}} \boldsymbol{K}_{k} \boldsymbol{U}_{k}\right] \\
\frac{\partial f_{1}}{\partial \boldsymbol{\kappa}} & =\frac{\boldsymbol{G}^{\mathrm{T}} \boldsymbol{H}^{\mathrm{T}}\left[\frac{\partial \overline{\tilde{\rho}}_{k}}{\partial \tilde{\rho}_{k}} V_{k}\right]}{V_{0}}
\end{aligned}
$$

where $V_{k}$ is the volume of element $k$ and $\lambda$ is the adjoint vector determined by $\boldsymbol{K} \boldsymbol{\lambda}=\boldsymbol{L}$. $\lambda_{k}, \boldsymbol{U}_{k}$ are the parts of $\lambda, \boldsymbol{U}$ corresponding to the nodal degrees of freedom of element $k$, $\boldsymbol{K}_{k}=\left(E_{0}-E_{\text {min }}\right) \boldsymbol{K}_{0} \approx E_{0} \boldsymbol{K}_{0} \cdot[$ [ ] represents $N_{\mathrm{e}} \times 1$ vectors whose elements obtained with $k$ ranging from 1 to $N_{\mathrm{e}} \cdot \partial \overline{\tilde{\rho}}_{k} / \partial \tilde{\rho}_{k}$ can be obtained from Eq. (6).

\subsection{Robust formulation}

In order to take different kinds of uncertainties into account, robust topology optimization methods have been developed (Beyer and Sendhoff 2007; Du and Sun 2017; Guo and Cheng 2010). For example, Kogiso et al. (2008) designed compliant mechanisms considering the uncertainty of the driving force direction. Chen et al. (2010) proposed a level-set based robust method considering the uncertainties of applied loads and material properties by a quadrature technique.

A simple formulation with dilated, intermediate and eroded designs was first proposed by Sigmund (2009) as a solution to improve structural robustness to uniform boundary variations in topology optimization. This idea was further improved and elaborated by Wang et al. (2011), revealing that this robust formulation can be an effective way to ensure local length scale to avoid one-node connected hinges and hence ensure distributed compliant hinges in the design of compliant mechanisms. On this basis, Schevenels et al. (2011) developed a probabilistic approach accounting for spatially varying manufacturing errors.

In this paper, following Wang et al. (2011), dilated, intermediate and eroded designs are formulated by utilizing the threshold projection with different parameters, e.g. $\eta=0.3, \eta=0.5$ and $\eta=0.7$. Then the original design problem (2) is reformulated into a min-max problem:

$$
\begin{gathered}
\min _{\boldsymbol{\kappa}}: \max \left\{f_{0}\left(\overline{\tilde{\boldsymbol{\rho}}}^{\mathrm{e}}\right), f_{0}\left(\overline{\tilde{\boldsymbol{\rho}}}^{\mathrm{i}}\right), f_{0}\left(\overline{\tilde{\boldsymbol{\rho}}}^{\mathrm{d}}\right)\right\} \\
\text { s.t. } \boldsymbol{K}\left(\overline{\tilde{\boldsymbol{\rho}}}^{\mathrm{e}}\right) \boldsymbol{U}^{\mathrm{e}}=\boldsymbol{P}, \\
\boldsymbol{K}\left(\overline{\tilde{\boldsymbol{\rho}}}^{\mathrm{i}}\right) \boldsymbol{U}^{\mathrm{i}}=\boldsymbol{P}, \\
\boldsymbol{K}\left(\overline{\tilde{\boldsymbol{\rho}}}^{\mathrm{d}}\right) \boldsymbol{U}^{\mathrm{d}}=\boldsymbol{P}, \\
f_{1}=\frac{\left(\overline{\tilde{\boldsymbol{\rho}}}^{\mathrm{d}}\right)^{\mathrm{T}} \boldsymbol{V}}{V_{0}} \leq \frac{V_{\mathrm{d}}^{*}}{V_{0}} \\
0 \leq \boldsymbol{\kappa} \leq 1 .
\end{gathered}
$$

Here, the volume fraction constraint is imposed on the dilated design and updated every 20 iterations to ensure the invariance of the volume of the intermediate design:

$V_{\mathrm{d}}^{*}=\frac{V^{*}}{V_{\mathrm{i}}} V_{\mathrm{d}}$

where $V^{*}$ is the prescribed value of the volume of intermediate design and $V_{\mathrm{i}}, V_{\mathrm{d}}$ are the current volume of the intermediate design and the dilated design.

\subsection{Flow chart of the design process}

The flow chart of the variable linking based robust topology optimization process can be seen in Fig. 3. The filtering and projection are applied on the whole domain instead of the single design cell. 


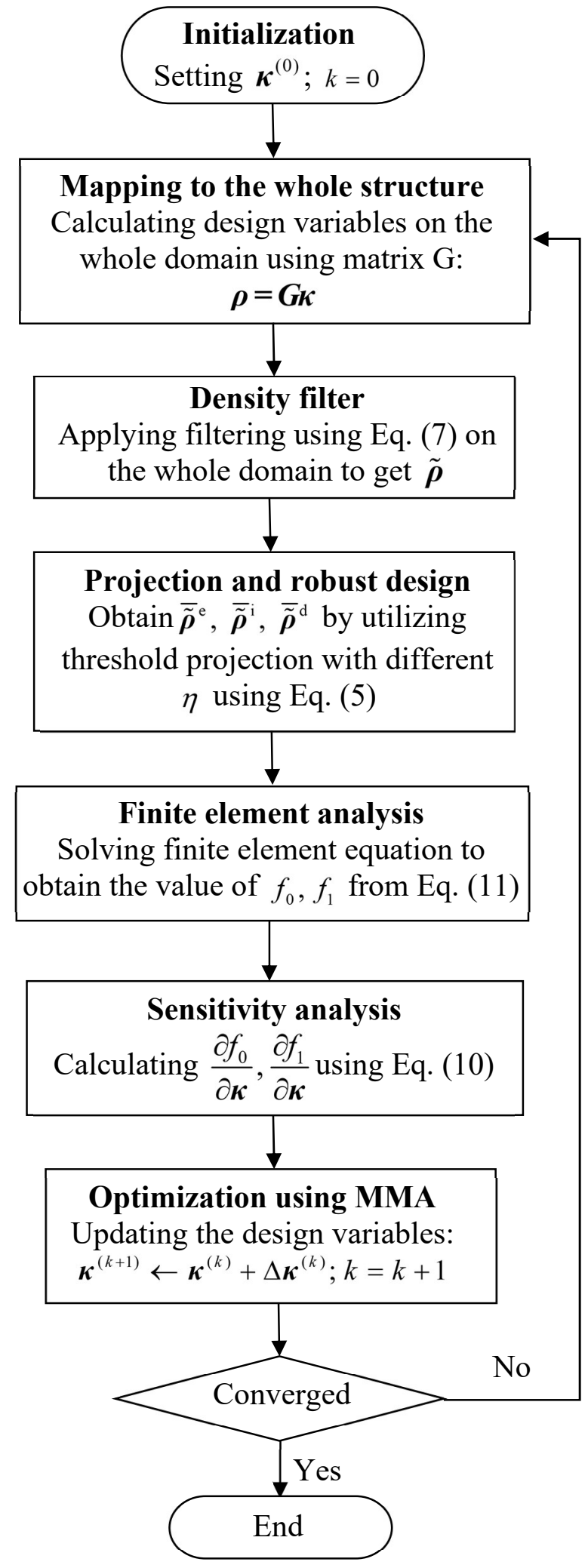

Fig. 3 Flow chart of topology optimization based on a variable linking scheme

\section{Numerical Examples}

\subsection{Example 1 - force inverter}

The force inverter is one of the most important benchmarks of compliant mechanisms (Sigmund 2007). A typical inverter design problem is shown in Fig. $4 a$. Due to symmetry, only the bottom half part of the structure is designed for faster computation as shown in Fig. $4 \mathrm{~b}$, which is supported at the bottom of the left edge with symmetry constraint on the upper boundary.

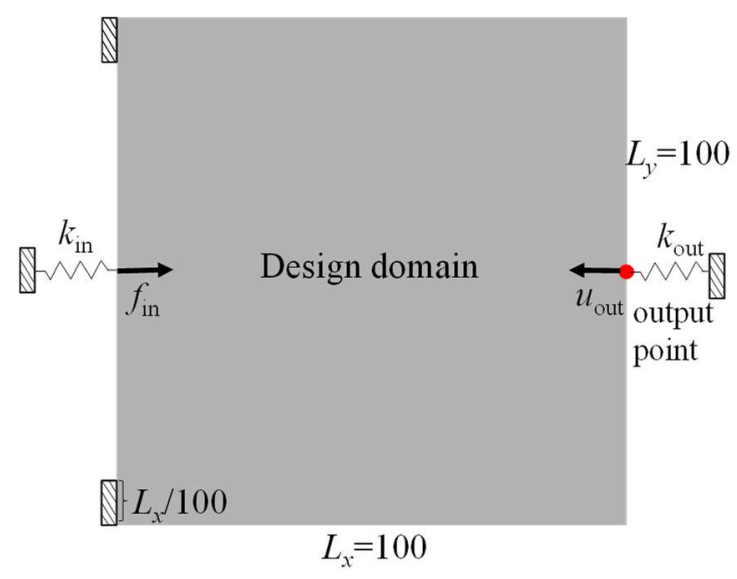

(a)

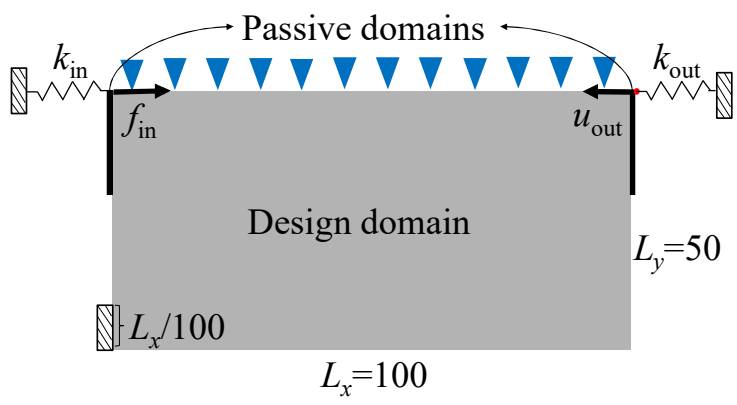

(b)

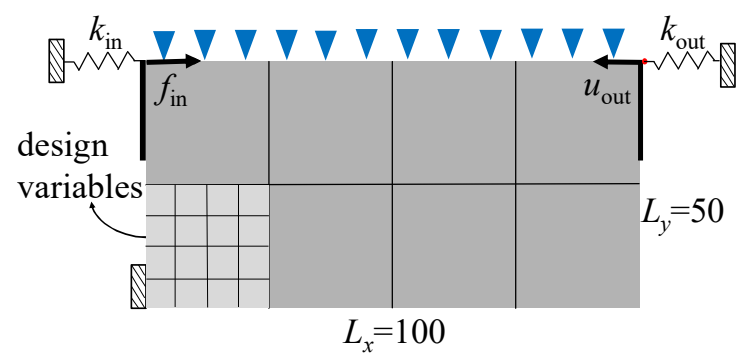

(c)

Fig. 4 a Design domain and boundary conditions for the force inverter. b Bottom half part with symmetry boundary condition. c Metamaterial force inverter design problem using a variable linking scheme. 
A regular rectangular discretization is employed in the FE model. Design variables are defined only on a single cell (Fig. 4c). The objective is to maximize the horizontal displacement $u_{\text {out }}$ of output point at the right side in the negative direction, for applied force $f_{\text {in }}=1$ at the left side and spring stiffnesses $k_{\text {in }}=1$ and $k_{\text {out }}=0.001$, respectively.

To ensure that the input loads can be transferred to the periodic structure, passive domains, i.e. black areas, are added at the input and output points. The length of the passive domains is set as four-fifths the length of one cell.

The volume fraction is $\alpha=0.3$, and the non-dimensional Young's modulus for the solid and the void phases are $E_{0}=1.0$ and $E_{\min }=10^{-9}$, respectively. The maximum iteration number is 400. A continuation method is adopted for the projection parameter $\beta$, i.e. starting from $\beta=1$, and the value of $\beta$ is doubled every 100 iterations.

The grey level of the obtained designs can be measured by an indicator proposed by Sigmund (2007):

$$
M_{\mathrm{nd}}=\frac{\sum_{j=1}^{N_{\mathrm{e}}} 4 \overline{\tilde{\rho}}_{j}\left(1-\overline{\tilde{\rho}}_{j}\right)}{N_{\mathrm{e}}} \times 100 \%
$$

The value of $M_{\text {nd }}$ may vary between $0 \%$ and $100 \%$, where " $0 \%$ " implies that the design has converged to absolute $0-1$, and " $100 \%$ " implies that all the elements have the same material volume density, i.e. $\overline{\tilde{\rho}}_{j}=0.5$.

Results with the robust formulation for different numbers of cells are shown in Fig. 5. The element resolution is $800 \times 400$ (320,000 elements) and the corresponding filter radius is set to be 3.1 times the element size. Parameters of MMA may need some slight adjustments to get more stable solutions in different cases.

It should be emphasized that our purpose is to find feasible metamaterial designs, rather than making continuous division of cells. Actually, further dividing the domain into more cells while keeping the element resolution unchanged, the design resolution in one cell may become too small to get a stable or physical solution.

The single cell design (see Fig. 5a) corresponds to the conventional compliant force inverter problem. As the number of cells increases, the displacement of the output point decreases (Tab. 1). Some preliminary explanations can be made as follows:

1. Compared to the conventional force inverter (i.e. the single cell case), metamaterial force inverters have lower transmission efficiency. Extra energy is dissipated as it is transferred between cells.

2. Due to computing limitations, the scale of the finite element mesh has an upper limit. For the metamaterial design, design freedom is reduced due to smaller number of design variables in each cell, which is a natural outcome of the variable linking scheme.

3. Structural redundancies can be observed on some of the metamaterial inverters. These redundancies are used to connect the periodic parts with the passive domains, but they become isolated parts inside the structure, which causes a waste of the base material. This issue occurs due to the need for satisfying boundary conditions, which is a natural drawback of the variable linking scheme.

4. Multi-cell designs can be seen as subsets of single cell design. The multi-cell configurations are also possible solutions of the single cell design problem, but not the opposite.

However, compared to conventional force inverters, metamaterial force inverters may provide better structural robustness, which will be discussed in Section 5. Besides, it can be noticed that the cellular configurations may vary slightly from each other since the variable linking scheme is applied using the design variables instead of the projected physical densities.

The structural deformations are shown in Fig. 6 to display more details on how the structures respond to the input force. 
In addition, as can be seen in Fig. 7, the three design realizations in the robust formulation have the same topology, but vary in feature size, representing over-, correctly, and under-etched structures respectively. There are no one-node connected hinges in the intermediate design, which indicates that the robust formulation also plays an important role in metamaterial mechanisms design.
It should be noted that the same length scale control (i.e. parameters of filtering, projection and robust formulation) is imposed independent of cell numbers, which inevitably results in rather localized hinges for cases with few cells (e.g. $2 \times 1,4 \times 2)$ and simpler cellular geometries with more distributed flexibilities for cases with more cells (e.g. $10 \times 5,16 \times 8)$.

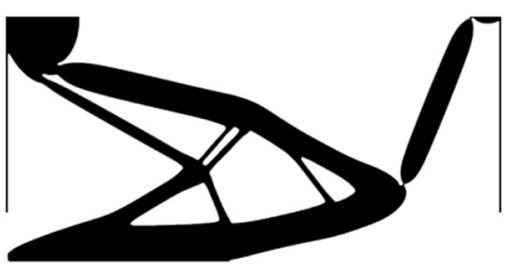

(a) $1 \times 1$ cell

Eroded design

$f_{0}=-2.400, f_{1}=0.291$

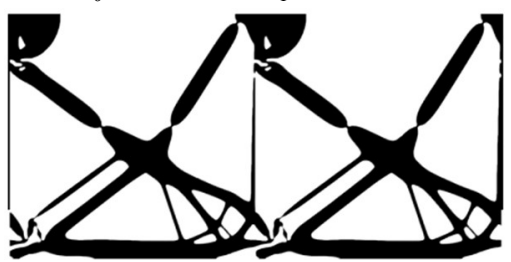

(b) $2 \times 1$ cells

Eroded design

$f_{0}=-1.124, f_{1}=0.282$

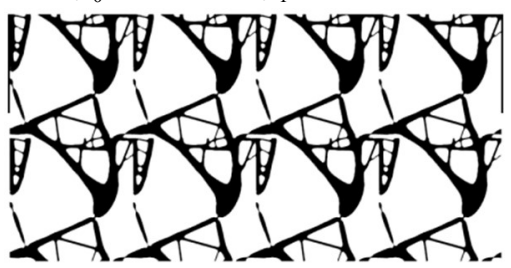

(c) $4 \times 2$ cells

Eroded design

$f_{0}=-0.246, f_{1}=0.262$

yny yry ynyryy

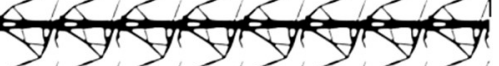

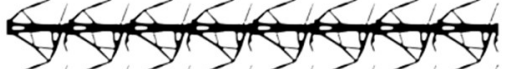
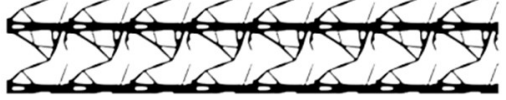

(d) $8 \times 4$ cells

Eroded design

$f_{0}=-0.214, f_{1}=0.236$

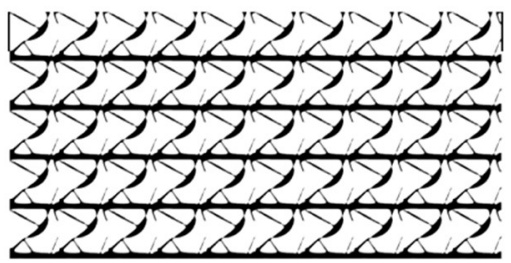

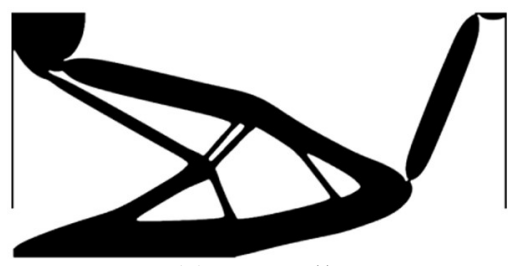

(a) $1 \times 1$ cell

Intermediate design

$f_{0}=-2.423, f_{1}=0.300$

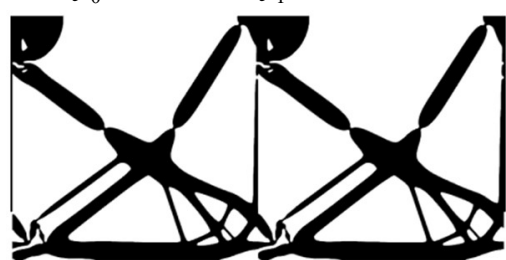

(b) $2 \times 1$ cells

Intermediate design

$f_{0}=-1.177, f_{1}=0.300$

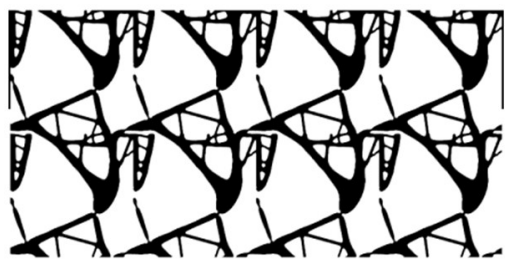

(c) $4 \times 2$ cells

Intermediate design

$f_{0}=-0.302, f_{1}=0.300$

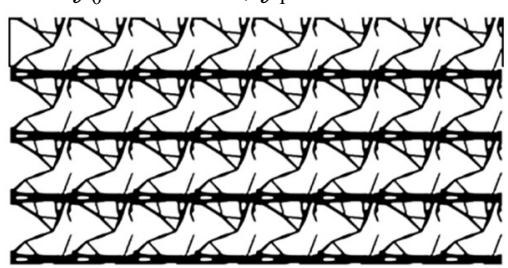

(d) $8 \times 4$ cells

Intermediate design

$f_{0}=-0.267, f_{1}=0.300$

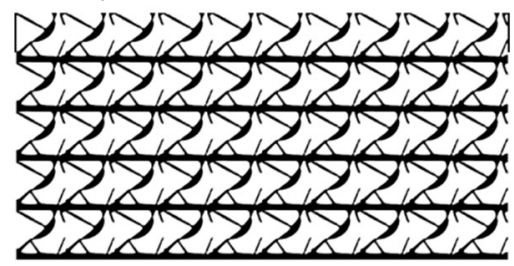

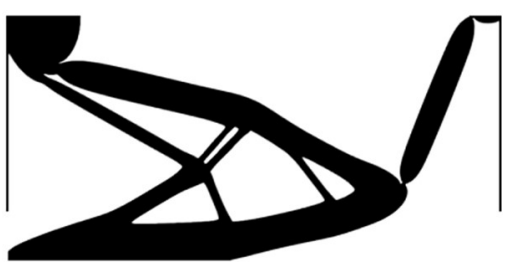

(a) $1 \times 1$ cell

Dilated design

$f_{0}=-2.399, f_{1}=0.311$

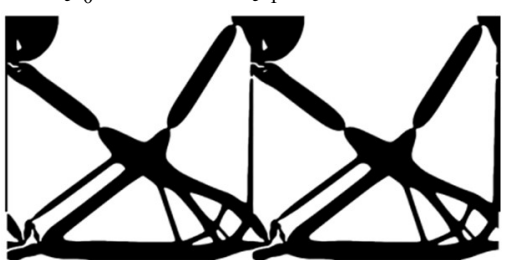

(b) $2 \times 1$ cells

Dilated design

$f_{0}=-1.122, f_{1}=0.318$

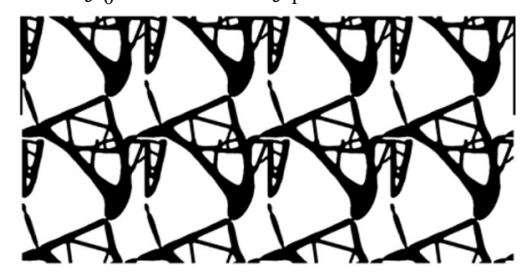

(c) $4 \times 2$ cells

Dilated design

$f_{0}=-0.249 f_{1}=0.336$

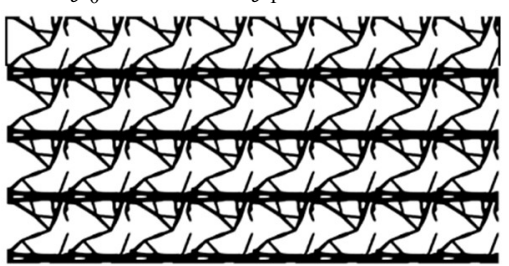

(d) $8 \times 4$ cells

Dilated design

$f_{0}=-0.215, f_{1}=0.359$

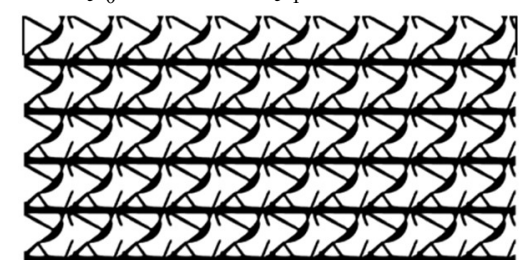


(e) $10 \times 5$ cells

Eroded design

$f_{0}=-0.130, f_{1}=0.234$

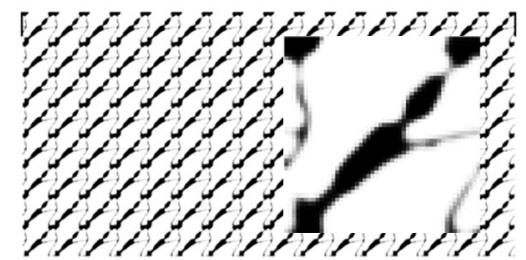

(f) $16 \times 8$ cells

Eroded design

$f_{0}=-0.001, f_{1}=0.191$ (e) $10 \times 5$ cells Intermediate design

$f_{0}=-0.242, f_{1}=0.300$

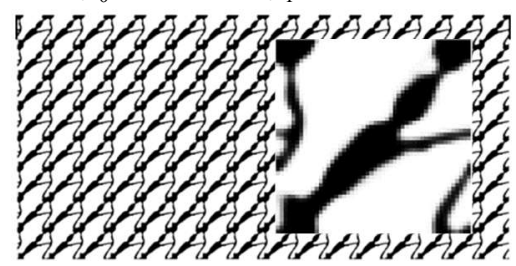

(f) $16 \times 8$ cells

Intermediate design

$f_{0}=-0.024, f_{1}=0.299$ (e) $10 \times 5$ cells

Dilated design

$f_{0}=-0.131, f_{1}=0.361$

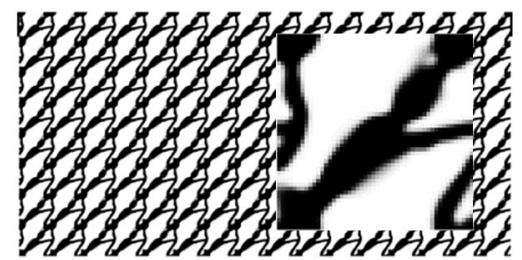

(f) $16 \times 8$ cells

Dilated design

$f_{0}=-0.001, f_{1}=0.407$

Fig. 5 Robust design of metamaterial force inverters with various cell numbers

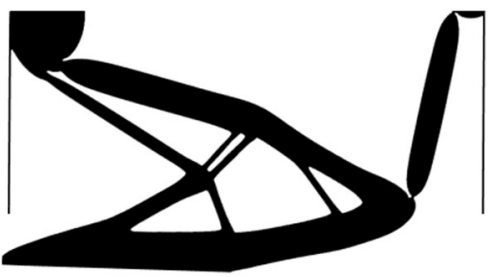

(a) $1 \times 1$ cell

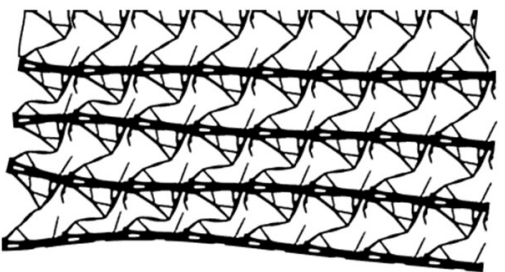

(d) $8 \times 4$ cells

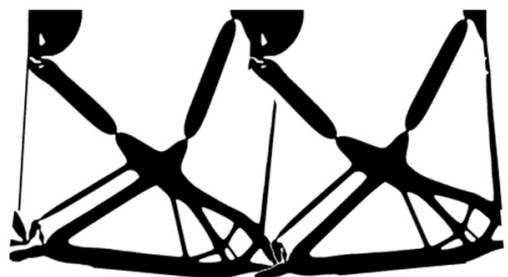

(b) $2 \times 1$ cells

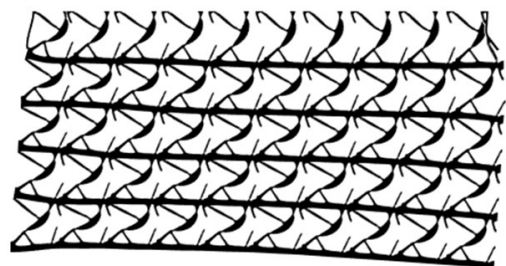

(e) $10 \times 5$ cells

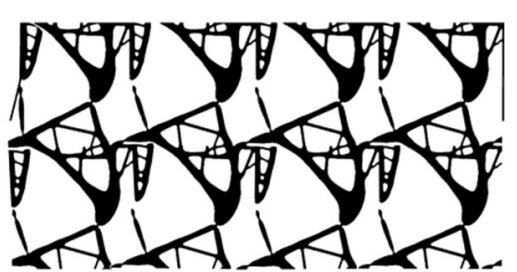

(c) $4 \times 2$ cells

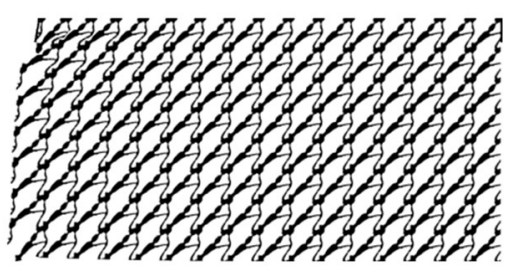

(f) $16 \times 8$ cells

Fig. 6 Deformation of force inverters with various cell numbers

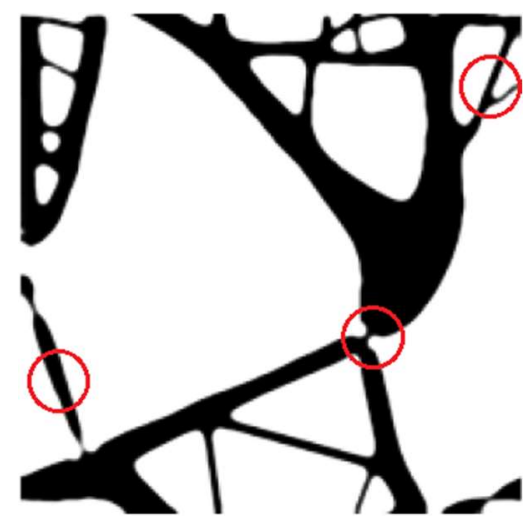

(a) Eroded design

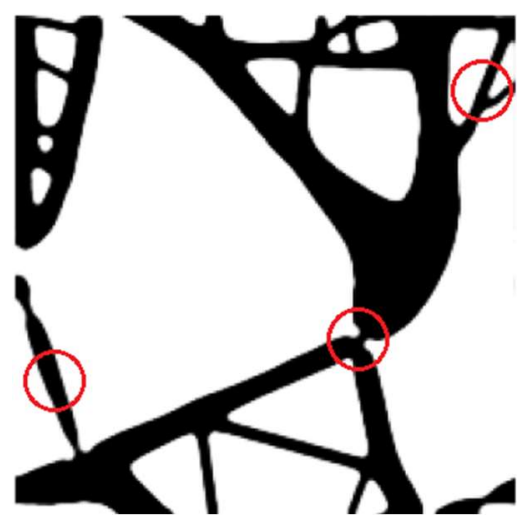

(b) Intermediate design

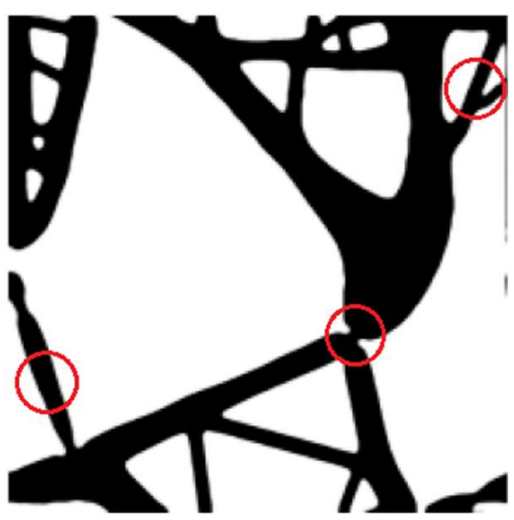

(c) Dilated design

Fig. 7 Cellular comparison between erode, intermediated, dilated designs $(4 \times 2$ cells $)$

Table 1 Comparisons of the intermediate designs for different cell numbers

\begin{tabular}{cccccc}
\hline $\begin{array}{c}\text { Element } \\
\text { resolution }\end{array}$ & $\begin{array}{c}\text { Number of } \\
\text { cells }\end{array}$ & Design space & $f_{0}$ & $f_{1}$ & $M_{n d}$ \\
\hline \multirow{2}{*}{$80 \times 400$} & $1 \times 1$ & $800 \times 400$ & -2.423 & 0.300 & $1.13 \%$ \\
& $2 \times 1$ & $400 \times 400$ & -1.177 & 0.300 & $2.04 \%$ \\
\hline
\end{tabular}




\begin{tabular}{rrrrc}
\hline $4 \times 2$ & $200 \times 200$ & -0.302 & 0.300 & $4.29 \%$ \\
$8 \times 4$ & $100 \times 100$ & -0.267 & 0.300 & $7.20 \%$ \\
$10 \times 5$ & $80 \times 80$ & -0.242 & 0.300 & $7.40 \%$ \\
$16 \times 8$ & $50 \times 50$ & -0.024 & 0.299 & $13.49 \%$ \\
\hline
\end{tabular}

\subsection{Inverters with multiple microstructures}

To introduce more flexibility in the design of metamaterial mechanisms, the design domain of Fig. 4 can be divided into multiple subdomains to be optimized separately with different cellular microstructures.

For example, as can be seen in Fig. 8, the variable linking scheme is now applied on the left and right part separately. Thus the amount of design variables is twice that of Fig. 4c. Otherwise, all the parameters remain the same as before.

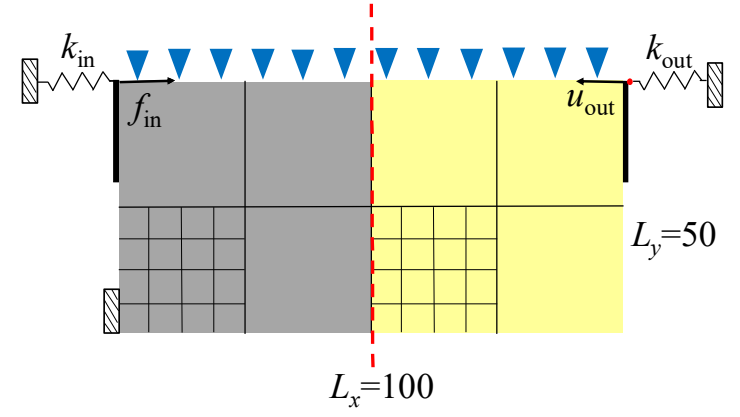

Fig. 8 Design domain for the force inverter with two subdomains

Results are shown in Fig. 9. The left part and the right part now converge to different cellular microstructures. Compared to results shown in Fig. 5, it is found that under the same cell division, results with two cellular microstructures have better mechanism performance than results with a single microstructure.

If we have more, e.g. four or eight subdomains to be optimized simultaneously, results with even better mechanism performance can be obtained. As can be seen in Fig. 10 and Fig. 11, allowing material to be taken away from some subdomains and concentrated to more rewarding areas, the design freedom is extended and the material utilization efficiency is thus improved a lot. Structural redundancies can still be observed in these cases. Obviously, when the number of subdomains go to infinity, the design will converge to the original single cell solution.

Fig. 12 displays the curves of objective values with respect to the total number of cells, different colors represent different amounts of cellular microstructures. In conclusion, the transmission efficiency (i.e. output displacement) decreases as the number of cell divisions increases. However, under the same number of cell division, structures with more kinds of cellular microstructures always have better mechanism performance than those with fewer microstructures.

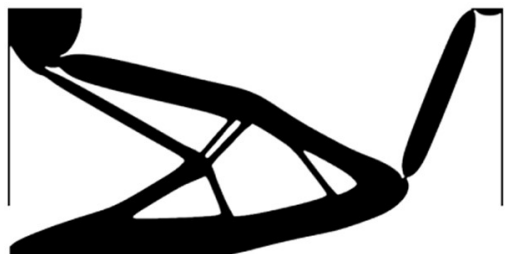

(a) $2 \times 1$ cells $f_{0}=-3.135, f_{1}=0.300$

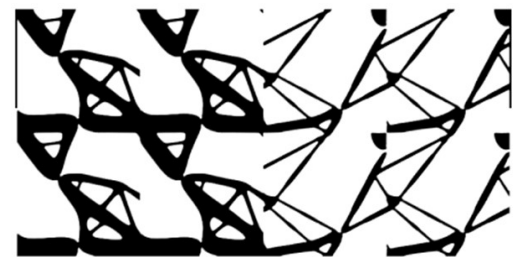

(b) $4 \times 2$ cells $f_{0}=-2.963, f_{1}=0.300$

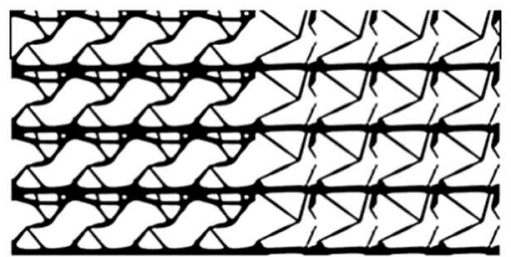

(c) $8 \times 4$ cells $f_{0}=-1.192, f_{1}=0.301$ 


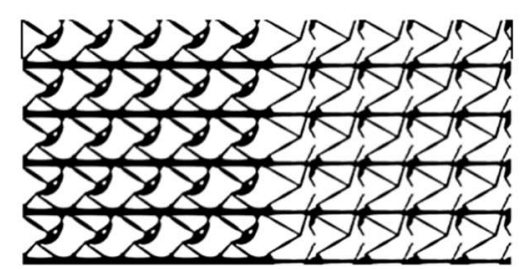

(d) $10 \times 5$ cells

$f_{0}=-0.779, f_{1}=0.299$

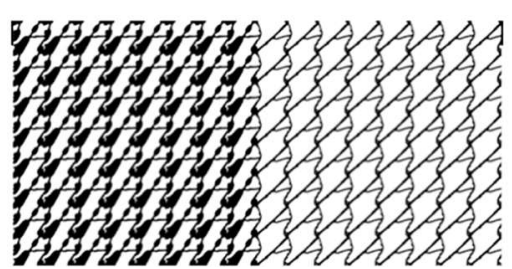

(e) $16 \times 8$ cells

$f_{0}=-0.471, f_{1}=0.299$

Fig. 9 Intermediate designs with various cell numbers (two subdomains)

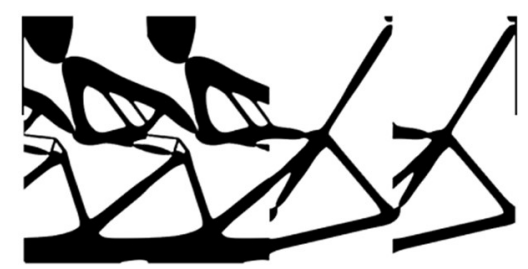

(a) $4 \times 2$ cells

$f_{0}=-3.135, f_{1}=0.300$

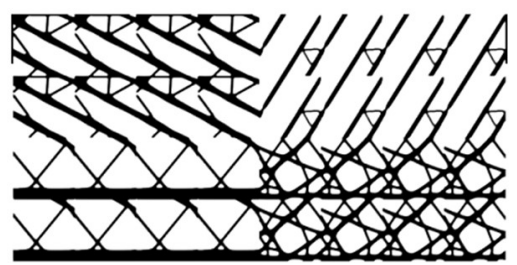

(b) $8 \times 4$ cells

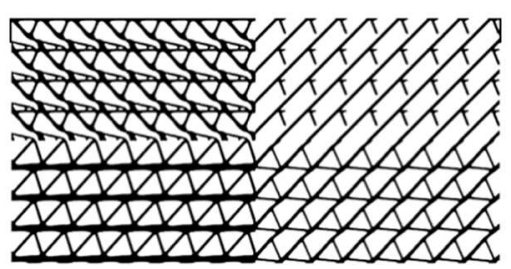

(c) $16 \times 8$ cells

Fig. 10 Intermediate designs with various cell numbers (four subdomains)

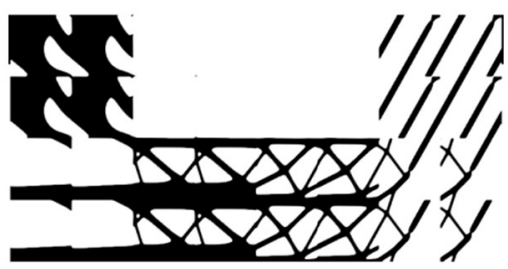

(a) $8 \times 4$ cells

$f_{0}=-3.135, f_{1}=0.300$

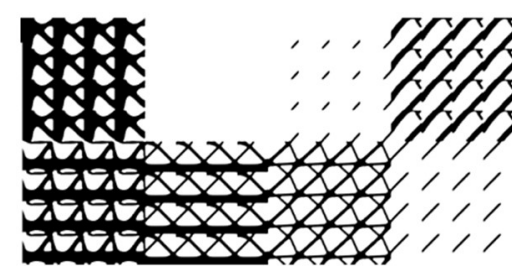

(b) $16 \times 8$ cells

$f_{0}=-2.963, f_{1}=0.300$

Fig. 11 Intermediate designs with various cell numbers (eight subdomains)

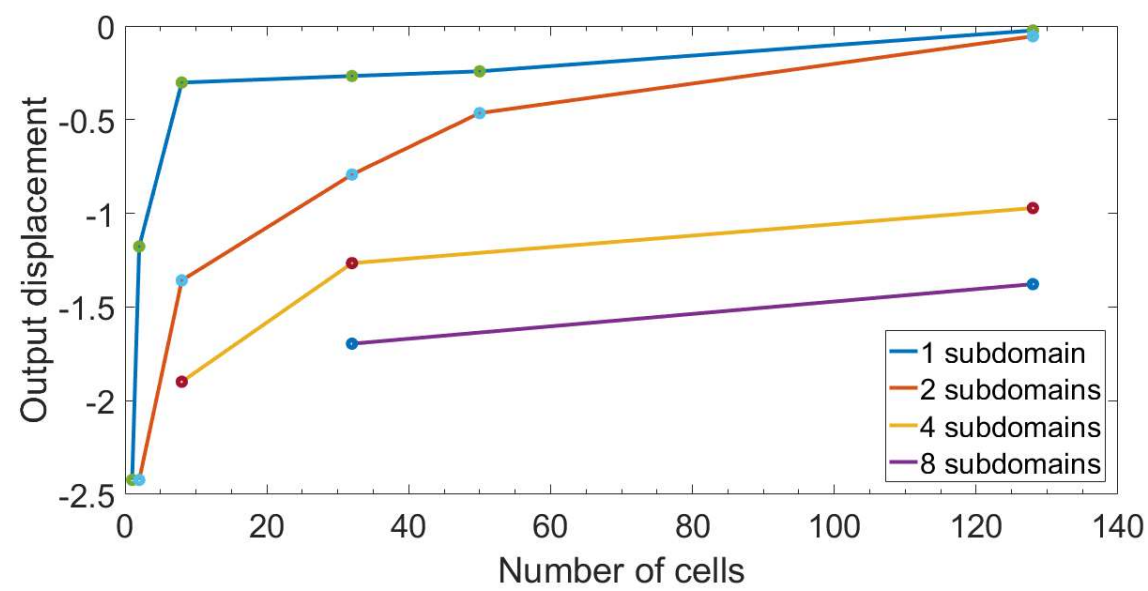

Fig. 12 Objective values of intermediate designs with respect to cell numbers

\subsection{Example 2 - asymmetric model}

The variable linking scheme is applicable to arbitrary shapes of design domains. For example, Fig. 13 sketches an irregular asymmetric design problem. Slip boundary conditions are imposed on the lower and right edges. Output displacement is defined orthogonal to the applied force.

The whole domain is divided into 240,000 elements. The length of the passive domains attached to the input and output region is set to two-fifths the length of one cell. The maximum iteration number is 300 . Other parameters remain the same as Example 1. 


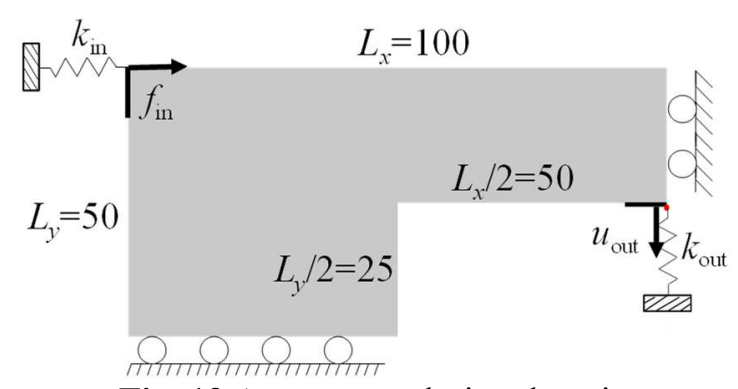

Fig. 13 Asymmetry design domain and Tab. 2. The multi-cell designs still have smaller output displacement than the singlecell design. What those designs have in common are ribs penetrating cells from top left to bottom right, which indicates how the input force is transmitted to the output point physically.

For metamaterial mechanisms, the imposed periodicity naturally results in boundaries having similar response as the output point.

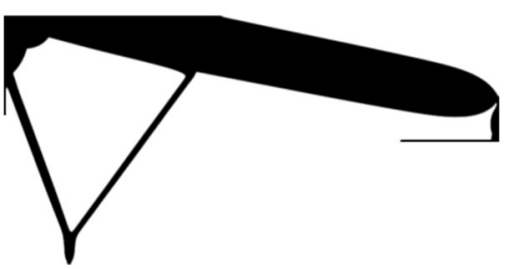

(a) $1 \times 1$ cell

$f_{0}=-2.980, f_{1}=0.300$

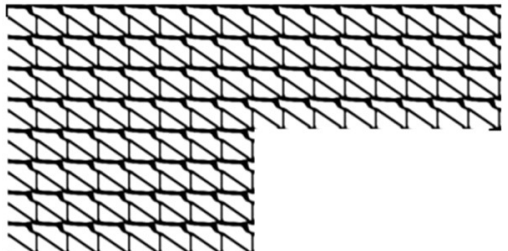

(d) $16 \times 8$ cells

$f_{0}=-0.771, f_{1}=0.299$

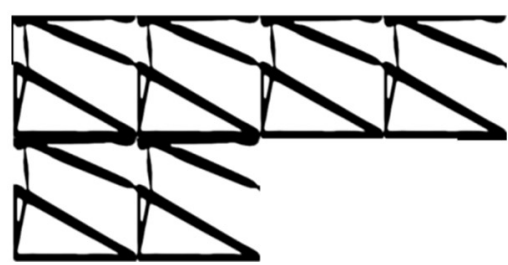

(b) $4 \times 2$ cells

$f_{0}=-1.504, f_{1}=0.300$

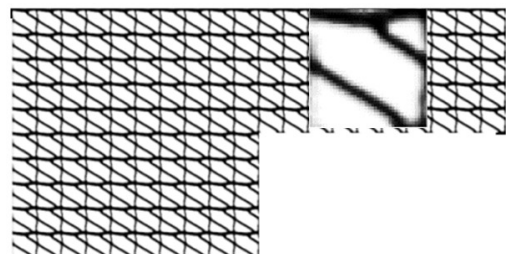

(e) $20 \times 10$ cells

$f_{0}=-0.682, f_{1}=0.291$

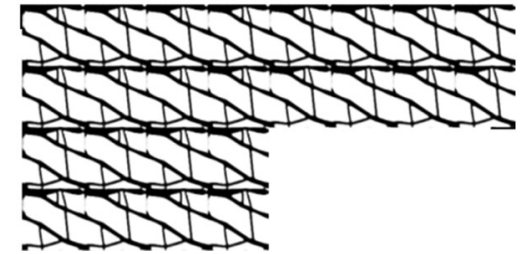

(c) $8 \times 4$ cells

$f_{0}=-1.047, f_{1}=0.299$

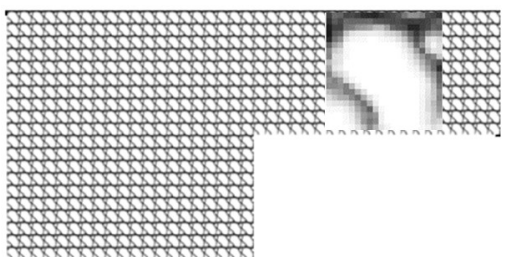

(f) $40 \times 20$ cells

$f_{0}=-0.294, f_{1}=0.247$

Fig. 14 Intermediate designs with various cell numbers (Maximize output in the negative direction)

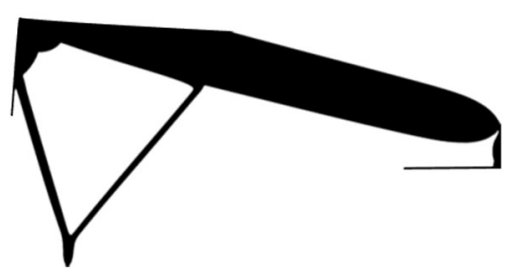

(a) $1 \times 1$ cell

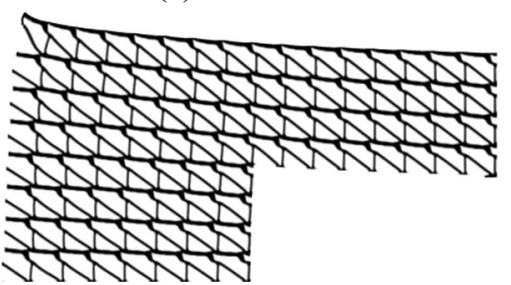

(d) $16 \times 8$ cells

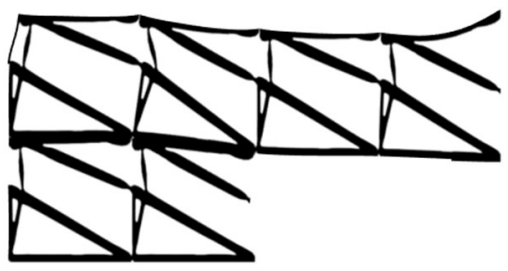

(b) $4 \times 2$ cells

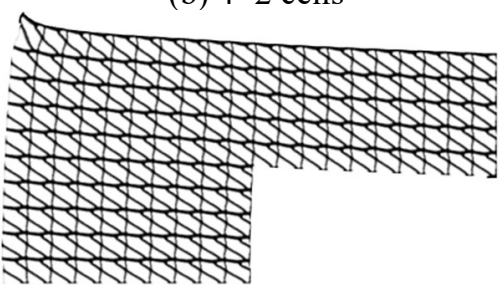

(e) $20 \times 10$ cells

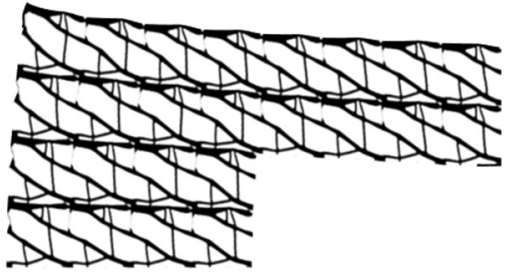

(c) $8 \times 4$ cells

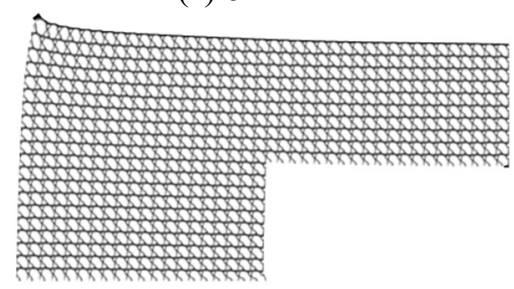

(f) $40 \times 20$ cells

Fig. 15 Deformation of intermediate designs with various cell numbers (negative direction)

Table 2 Comparisons of the intermediate designs for different cell numbers

\begin{tabular}{cccccc}
\hline Type & $\begin{array}{c}\text { Number of } \\
\text { cells }\end{array}$ & Design space & $f_{0}$ & $f_{1}$ & $M_{n d}$ \\
\hline Uniform design & -- & -- & -0.066 & 0.300 & $84 \%$
\end{tabular}




\begin{tabular}{cccccc} 
& $1 \times 1$ & 240000 & -2.980 & 0.300 & $0.79 \%$ \\
& $4 \times 2$ & $200 \times 200$ & -1.504 & 0.300 & $1.84 \%$ \\
$\begin{array}{c}\text { Metamaterial } \\
\text { designs }\end{array}$ & $8 \times 4$ & $100 \times 100$ & -1.047 & 0.299 & $4.23 \%$ \\
& $16 \times 8$ & $50 \times 50$ & -0.771 & 0.299 & $5.16 \%$ \\
& $20 \times 10$ & $40 \times 40$ & -0.682 & 0.291 & $7.65 \%$ \\
& $40 \times 20$ & $20 \times 20$ & -0.294 & 0.247 & $11.90 \%$ \\
\hline
\end{tabular}

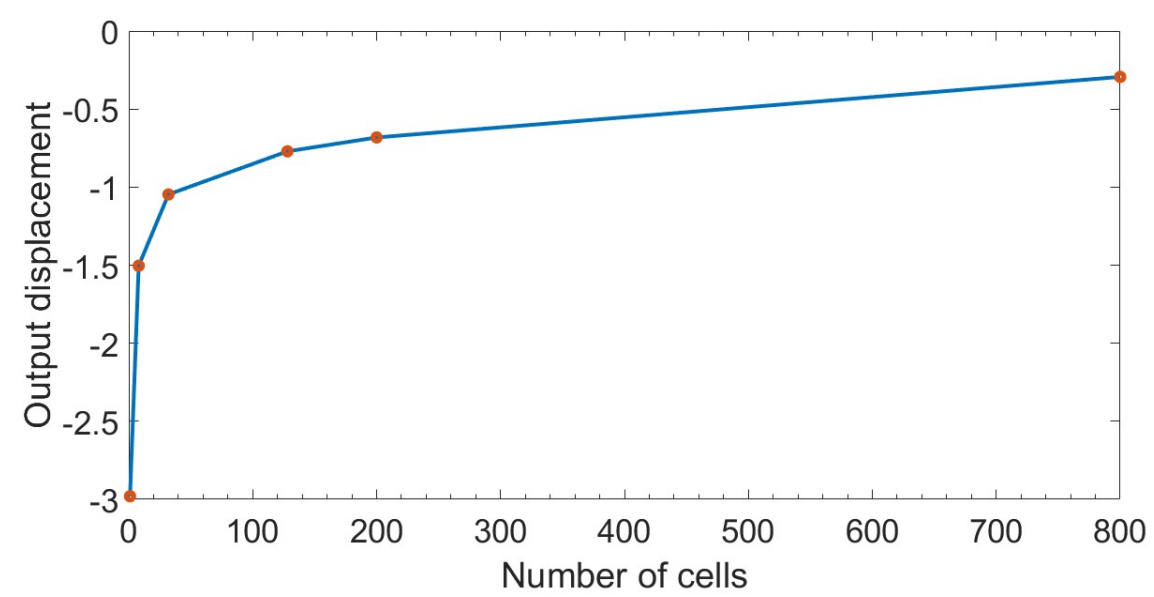

Fig. 16 Objective values of intermediate designs with respect to cell numbers

\section{Structural Robustness Analysis}

\subsection{Local damage tolerance}

Conventional compliant mechanisms obtained through the robust formulation are robust to manufacturing uncertainties, but when it comes to local defects, metamaterial mechanisms are expected to have better structural robustness since the failure of some cells may not lead to the failure of the whole structure.

To verify this, a simplified local damage evaluation (Jansen et al. 2014; Wu et al. 2018) is employed to examine structural robustness when local failure happens due to e.g. collision, explosion or manufacturing error.

It is assumed that a quadrilateral region with fixed size is damaged and it may appear arbitrarily. The force inverters obtained in Section 4.1 are used as test cases. As can be seen in Fig. 17, materials in the red squares are removed to simulate damage. The dimension of the square is $0.1 L_{y}$.

Here we first fix the horizontal coordinates of the damaged region (i.e. the lower-left corner is at four fifths of the long side) and change its vertical position gradually. As can be seen in Fig. 18, the single cell design totally fails when defects occur on its key hinges, while metamaterial inverters exhibit smaller variations of the output displacements, which implies that they are less sensitive to the prescribed damage appearing in the specific domain and hence have better structural robustness.

Next we change the horizontal and vertical position of the damaged region simultaneously. For each case, the horizontal position changes from 0 to $0.9 L_{x}$, with increments of $0.1 L_{x}$, and the vertical position changes from 0 to $0.9 L_{y}$, with increments of $0.05 L_{y}$, the total number of tests with different positions of damage is 190 for each case. 
Comparison of structural robustness among different designs is shown in Tab. 3. The conventional force inverter, i.e. the single cell case has the highest possibility of transmission efficiency loss over $10 \%$ and $50 \%$. Additionally, standard deviation and root mean square error (RMSE) is also used to evaluate the data dispersion. Statistically, it proves that metamaterial inverters have better local damage tolerance than the conventional force inverter.

Now we expand the local damage area, e.g. quadruple the damaged area by setting the dimension of the square to $0.2 L_{y}$. Again, changing the horizontal position of the damaged region from 0 to $0.8 L_{x}$, with increments of $0.1 L_{x}$, vertical position from 0 to $0.8 L_{y}$, with increments of $0.05 L_{y}$. Comparison of robustness among different designs are shown in Tab. 4. The advantage of metamaterial mechanism designs is even more pronounced in this case.

\subsection{Stress analysis}

To make further comparisons between conventional compliant inverters and metamaterial inverters, stress analysis is performed in ANSYS.

Fig. 19 displays the von Mises stress distributions on the design domain of the three typical designs: $1 \times 1,8 \times 4,10 \times 5$. The material elastic modulus, input force and spring stiffness are all magnified by a factor of $2.06 \times 10^{5}$, this kind of scaling is feasible since the analysis is limited to linear elasticity. The maximum value of the stress colorbar is set to 6000 in order to facilitate comparison.

For the single cell design, the maximum stress appears on the fixed constraint boundary at the lower left corner, and there are obvious stress concentration on these localized hinges. But for multi-cell designs, there are no obvious stress concentrations over the whole structure.

Tab. 5 lists the value of extreme stresses for the three cases. The maximum stress for the single cell case is much bigger than the others.

In conclusion, compared to conventional compliant mechanisms, metamaterial mechanisms have more uniform stress distribution, with rare stress concentrations. One reason for this is that due to the same length scale control imposed, designs with many cells obtained are distributed mechanisms, while single-cell design still have more localized hinges.

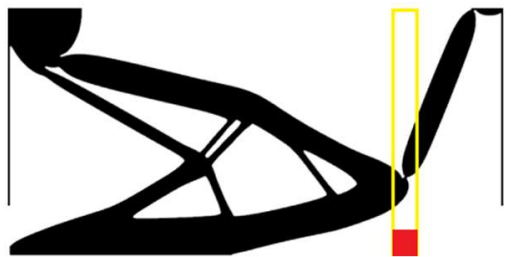

(a) $1 \times 1$ cell

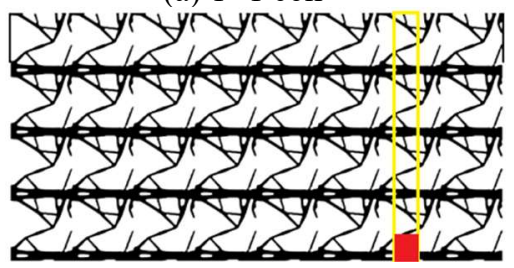

(d) $8 \times 4$ cells

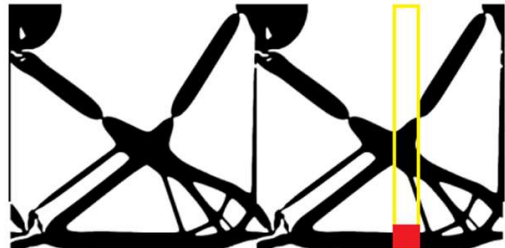

(b) $2 \times 1$ cells

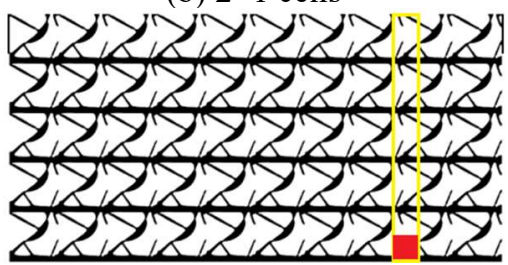

(e) $10 \times 5$ cells

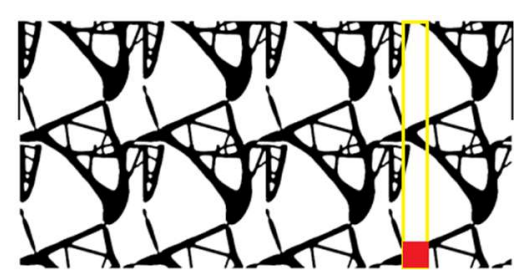

(c) $4 \times 2$ cells

Fig. 17 Illustration of local damage

Table 3 Comparisons of structural robustness for different cell numbers

\begin{tabular}{ccccc}
\hline $\begin{array}{c}\text { Number of } \\
\text { cells }\end{array}$ & $\begin{array}{c}\text { Probability of } \\
\text { efficiency loss } \\
\text { over } 10 \%\end{array}$ & $\begin{array}{c}\text { Probability of } \\
\text { efficiency loss } \\
\text { over } 50 \%\end{array}$ & $\begin{array}{c}\text { Standard } \\
\text { Deviation }\end{array}$ & $\begin{array}{c}\text { Root Mean } \\
\text { Square Error* }\end{array}$ \\
\hline
\end{tabular}




\begin{tabular}{ccccc}
\hline $1 \times 1$ & $17.89 \%$ & $5.79 \%$ & 0.5194 & 0.5533 \\
$2 \times 1$ & $5.26 \%$ & $4.21 \%$ & 0.3587 & 0.4126 \\
$4 \times 2$ & $4.21 \%$ & $4.21 \%$ & 0.1051 & 0.1185 \\
$8 \times 4$ & $4.21 \%$ & $4.21 \%$ & 0.0738 & 0.0999 \\
$10 \times 5$ & $4.21 \%$ & $4.21 \%$ & 0.0609 & 0.1315 \\
$16 \times 8$ & $3.68 \%$ & $3.68 \%$ & 0.0138 & 0.0152 \\
\hline
\end{tabular}

* The reference value of RMSE for each design is its corresponding optimal value.

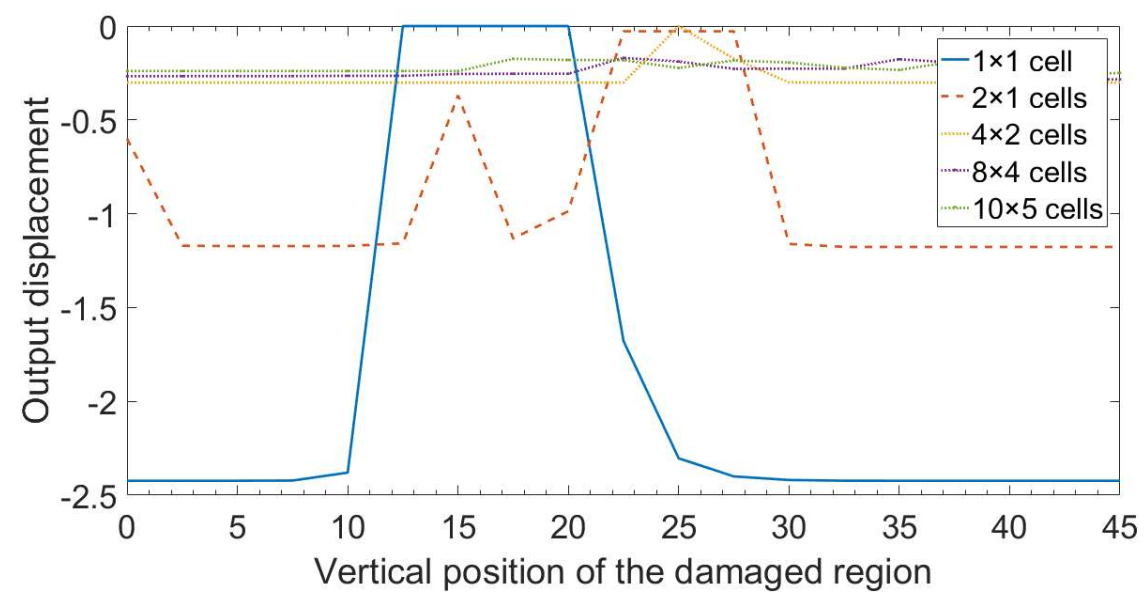

Fig. 18 Output displacements of force inverters with respect to a constantly vertically relocated damage region

Table 4 Comparisons of structural robustness for different cell numbers (damage area expanded)

\begin{tabular}{ccccc}
\hline $\begin{array}{c}\text { Number of } \\
\text { cells }\end{array}$ & $\begin{array}{c}\text { Probability of } \\
\text { efficiency loss } \\
\text { over 10\% }\end{array}$ & $\begin{array}{c}\text { Probability of } \\
\text { efficiency loss } \\
\text { over 50\% }\end{array}$ & $\begin{array}{c}\text { Standard } \\
\text { Deviation }\end{array}$ & $\begin{array}{c}\text { Root Mean } \\
\text { Square Error }\end{array}$ \\
\hline $1 \times 1$ & $54.25 \%$ & $27.45 \%$ & 0.8689 & 1.1152 \\
$2 \times 1$ & $22.22 \%$ & $16.34 \%$ & 0.4486 & 0.6543 \\
$4 \times 2$ & $12.42 \%$ & $12.42 \%$ & 0.1427 & 0.1937 \\
$8 \times 4$ & $12.42 \%$ & $12.42 \%$ & 0.0925 & 0.1707 \\
$10 \times 5$ & $12.42 \%$ & $12.42 \%$ & 0.0655 & 0.0940 \\
$16 \times 8$ & $12.42 \%$ & $12.42 \%$ & 0.0224 & 0.0263 \\
\hline
\end{tabular}




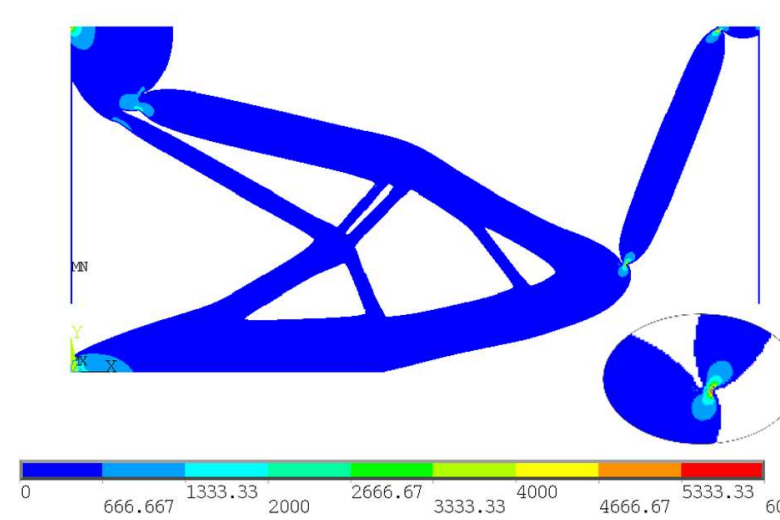

(a) $1 \times 1$

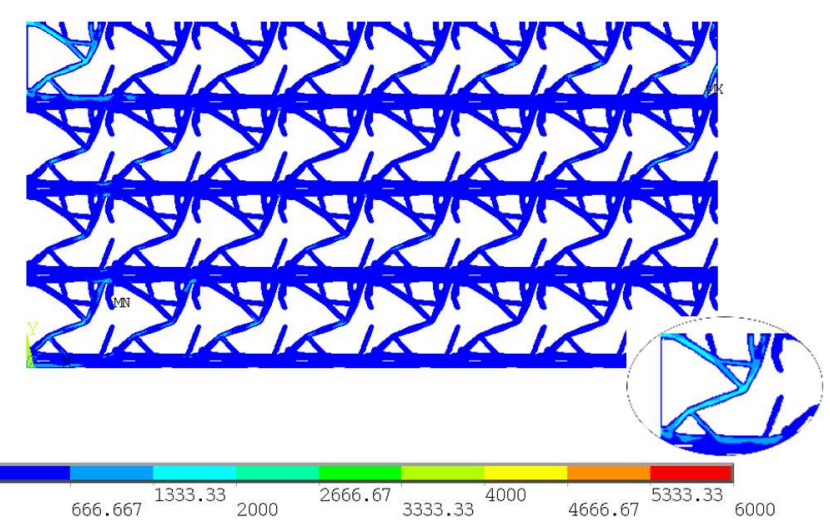

(b) $8 \times 4$
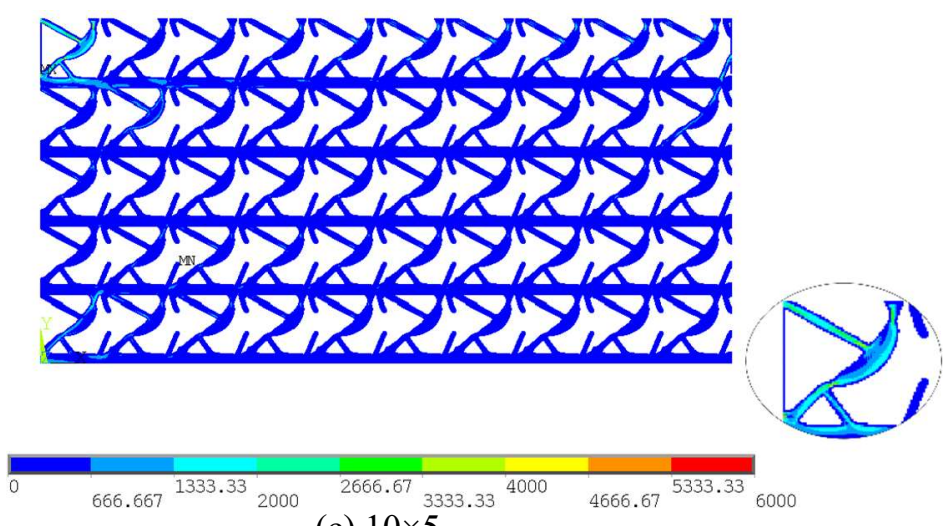

(c) $10 \times 5$

Fig. 19 Stress distributions over the three cases of force inverters

Table 5 Extreme stresses for the three cases

\begin{tabular}{cc}
\hline Number of cells & Maximum stress \\
\hline $1 \times 1$ & 16789.2 \\
$8 \times 4$ & 5878.8 \\
$10 \times 5$ & 8218.2 \\
\hline
\end{tabular}




\section{Conclusions}

This paper proposes a variable linking method for the topological design of metamaterial mechanism, which is a relatively new concept. Metamaterial mechanisms are composed of macroscopic cells, thus there is no need or use to introduce homogenization method that require separation of scales.

Metamaterial mechanisms have both pros and cons. Compared to conventional compliant mechanisms, metamaterial mechanisms have lower force transmission efficiency (i.e. output displacement). Some possible reasons for this, including structural redundancies, have been discussed in detail.

An advantage of metamaterial mechanisms is that when considering possible local physical defects or damage, the single cell design will have a high probability of systematic functional failure, while the robustness of multi-cell design is much better.

The best design and use of metamaterial mechanisms with suitable number of cell divisions should be decided by a trade-off between the transmission efficiency and structural robustness, while considering the manufacturing accuracy.

On the other hand, metamaterial mechanisms may generate the output or impose the input on the whole boundary of the structural domain, which will bring great flexibility to assembly and to adapt to the multi input/output and even multi-functional requirements.

Multiple subdomains can be adopted to introduce more flexibility in the design of metamaterial mechanisms. Compared to designs with a single cellular microstructure, designs with multiple cellular microstructures always display better mechanism performances.

Another possible way to further improve the behaviour of metamaterial mechanism is to do bi-level topology optimization by optimizing the base cell's topological configuration and the cell distribution over the whole design domain simultaneously. Besides, nonlinear theory can also be considered in future research.

Acknowledgement The research is supported by NSFC (Grant No. 11772170), the Key Laboratory of Spacecraft Design Optimization and Dynamic Simulation Technologies (Beihang University), Ministry of Education, China under Grant No. 2019KF001, Tsinghua University Tutor Research Fund, and the Project on Electric Driver Seat Technology for Large Passenger Aircraft (Grant No. MJ-2018-S-44), and the Villum Investigator Project InnoTop by the Villum Foundation, which are gratefully acknowledged by the authors. In addition, the authors sincerely acknowledge Dr. Pingzhang Zhou's help with the basic MATLAB code.

\section{Compliance with ethical standards}

Conflict of interest The authors declare that they have no conflict of interest.

Replication of results All the datasets in this paper are generated using our homemade MATLAB codes. The full datasets, and the source codes, can be available only for academic use from the corresponding author with reasonable request.

\section{References}

Ananthasuresh G, Kota S, Gianchandani Y (1994) A methodical approach to the design of compliant micromechanisms. Solid-State Sensor and Actuator Workshop:189-192

Bauer J, Schroer A, Schwaiger R, Kraft O (2016) Approaching theoretical strength in glassy carbon nanolattices. Nature Materials 15:438-443 doi:10.1038/nmat4561

Bendsøe M, Sigmund O (1999) Material interpolation schemes in topology optimization Archive of Applied Mechanics 69:635-654 doi: $10.1007 / \mathrm{s} 004190050248$

Bendsøe MP, Sigmund O (2004) Topology Optimization: Theory, Methods and Applications. Springer Press, Berlin

Beyer H-G, Sendhoff B (2007) Robust optimization A comprehensive survey Computer Methods 
in Applied Mechanics and Engineering 196:3190-3218

Chen S, Chen W, Lee S (2010) Level set based robust shape and topology optimization under random field uncertainties Structural and Multidisciplinary Optimization 41:507-524 doi:10.1007/s00158-009-0449-2

Chen W, Huang X (2019) Topological design of 3D chiral metamaterials based on couple-stress homogenization. Journal of the Mechanics and Physics of Solids 131:372-386 doi:https://doi.org/10.1016/j.jmps.2019.07.0 $\underline{14}$

Christiansen RE, Sigmund O (2016a) Designing meta material slabs exhibiting negative refraction using topology optimization Structural and Multidisciplinary Optimization 54:469-482 doi:10.1007/s00158-016-1411-8

Christiansen RE, Sigmund O (2016b) Experimental validation of systematically designed acoustic hyperbolic meta material slab exhibiting negative refraction Applied Physics Letters 109 doi:10.1063/1.4962441

Christiansen RE, Wang F, Sigmund O (2019) Topological Insulators by Topology Optimization Phys Rev Lett 122:234502 doi:10.1103/PhysRevLett.122.234502

Clausen A, Wang F, Jensen JS, Sigmund O, Lewis JA (2015) Topology Optimized Architectures with Programmable Poisson's Ratio over Large Deformations Advanced Materials 27:5523-5527

da Silva GA, Beck AT, Sigmund O (2019) Topology optimization of compliant mechanisms with stress constraints and manufacturing error robustness Computer Methods in Applied Mechanics and Engineering 354:397-421 doi:https://doi.org/10.1016/j.cma.2019.05.04 6

De Leon DM, Alexandersen J, O. Fonseca JS, Sigmund O (2015) Stress-constrained topology optimization for compliant mechanism design Structural and Multidisciplinary Optimization 52:929-943 doi:10.1007/s00158-015-1279-z

Diaz AR, Sigmund O (2009) A topology optimization method for design of negative permeability metamaterials. Structural Multidisciplinary Optimization 41:163-177

Du J, Sun C (2017) Reliability-based vibro-acoustic microstructural topology optimization Structural and Multidisciplinary Optimization 55:1195-1215 doi:10.1007/s00158-016-1571-6

Florijn B, Coulais C, van Hecke M (2014) Programmable Mechanical Metamaterials. Physical Review Letters 113:175503 doi:10.1103/PhysRevLett.113.175503

Frenzel T, Kadic M, Wegener M (2017) Three- dimensional mechanical metamaterials with
a twist. Science 358:1072-1074 doi:10.1126/science.aao4640

Guo X, Cheng G (2010) Recent development in structural design and optimization. Acta Mechanica Sinica:19-35

Guo X, Zhang W, Zhang L (2013) Robust structural topology optimization considering boundary uncertainties Computer Methods in Applied Mechanics and Engineering 253:356-368 doi:https://doi.org/10.1016/j.cma.2012.09.00 $\underline{5}$

Ion A, Frohnhofen J, Wall L, Kovacs R Metamaterial Mechanisms. In: Proceedings of UIST'16, 2016.

Jansen M, Lombaert G, Schevenels M (2015) Robust topology optimization of structures with imperfect geometry based on geometric nonlinear analysis Computer Methods in Applied Mechanics and Engineering 285:452-467

Jansen M, Lombaert G, Schevenels M, Sigmund O (2014) Topology optimization of fail-safe structures using a simplified local damage model Structural and Multidisciplinary Optimization 49:657-666 doi:10.1007/s00158-013-1001-y

Kogiso N, Ahn W, Nishiwaki S, Izui K, Yoshimura M (2008) Robust Topology Optimization for Compliant Mechanisms Considering Uncertainty of Applied Loads Journal of Advanced Mechanical Design Systems and Manufacturing - J ADV MECH DES SYST MANUF 2:96-107 doi:10.1299/jamdsm.2.96

Lakes R (1987) Foam Structures with a Negative Poisson's Ratio. Science 235:1038-1040 doi:10.1126/science.235.4792.1038

Lakes RS, Lee T, Bersie A, Wang YC (2001) Extreme damping in composite materials with negative-stiffness inclusions Nature 410:565567 doi: $10.1038 / 35069035$

Lazarov B, Schevenels M, Sigmund O (2011) Robust design of large-displacement compliant mechanisms Mechanical Sciences 2:175-182 doi:10.5194/ms-2-175-2011

Lazarov BS, Schevenels M, Sigmund O (2012a) Topology optimization considering material and geometric uncertainties using stochastic collocation methods Structural and Multidisciplinary Optimization 46:597-612 doi:10.1007/s00158-012-0791-7

Lazarov BS, Schevenels M, Sigmund O (2012b) Topology optimization with geometric uncertainties by perturbation techniques 90:1321-1336 doi:10.1002/nme.3361

Lee E, Gea HC (2014) A strain based topology optimization method for compliant mechanism design Structural and Multidisciplinary Optimization 49:199-207 
doi:10.1007/s00158-013-0971-0

Lu L, Yamamoto T, Otomori M, Yamada T, Nishiwaki S (2013) Topology optimization of an acoustic metamaterial with negative bulk modulus using local resonance. Finite Elements in Analysis Design 72:1-12

Milton GW (1992) Composite materials with poisson's ratios close to -1 . Journal of the Mechanics and Physics of Solids 40:1105-1137 doi:https://doi.org/10.1016/00225096(92)90063-8

Nicolaou ZG, Motter AE (2012) Mechanical metamaterials with negative compressibility transitions Nature Materials 11:p.608-613

Nørgaard S, Sigmund O, Lazarov B (2016) Topology optimization of unsteady flow problems using the lattice Boltzmann method Journal of Computational Physics 307:291-307 doi:10.1016/j.jcp.2015.12.023

Schevenels M, Lazarov BS, Sigmund O (2011) Robust topology optimization accounting for spatially varying manufacturing errors Computer Methods in Applied Mechanics and Engineering 200:3613-3627 doi:https://doi.org/10.1016/j.cma.2011.08.00 $\underline{6}$

Sigmund O (1994) Materials with prescribed constitutive parameters: An inverse homogenization problem. International Journal of Solids and Structures 31:23132329

Sigmund O (1997) On the design of compliant mechanisms using topology optimization. Mechanics of Structures and Machines 25:493-524

Sigmund O (2007) Morphology-based black and white filters for topology optimization Structural and Multidisciplinary Optimization 33:401424 doi:10.1007/s00158-006-0087-x
Sigmund O (2009) Manufacturing tolerant topology optimization. Acta Mechanica Sinica (02):83-95

Sigmund O, Torquato S (1996) Composites with extremal thermal expansion coefficients Applied Physics Letters 69

Svanberg K (1987) The method of moving asymptotes - a new method for structural optimization. Int $\mathrm{J}$ Numer Meth Engng 24:359-373 doi:10.1002/nme.1620240207

Wang F (2018) Systematic design of 3D auxetic lattice materials with programmable Poisson's ratio for finite strains Journal of the Mechanics \& Physics of Solids:S0022509617308438

Wang F, Lazarov BS, Sigmund O (2011) On projection methods, convergence and robust formulations in topology optimization. Structural Multidisciplinary Optimization 43:767-784

Wu J, Aage N, Westermann R, Sigmund O (2018) Infill Optimization for Additive Manufacturing-Approaching Bone-Like Porous Structures IEEE Transactions on Visualization and Computer Graphics 24:1127-1140 doi:10.1109/TVCG.2017.2655523

Yoon GH, Kim YY, Bendsøe MP, Sigmund O (2004) Hinge-free topology optimization with embedded translation-invariant differentiable wavelet shrinkage Structural and Multidisciplinary Optimization 27:139-150 doi:10.1007/s00158-004-0378-z

Zhu B, Zhang X, Zhang H, Liang J, Zang H, Li H, Wang R (2020) Design of compliant mechanisms using continuum topology optimization: A review Mechanism and Machine Theory 143:103622 doi:https://doi.org/10.1016/j.mechmachtheor y.2019.103622 